\title{
Research Article \\ Connecting Fuzzifying Topologies and Generalized Ideals by Means of Fuzzy Preorders
}

\author{
G. Elsalamony \\ Department of Mathematics, Faculty of Science, Benha University, Benha, Egypt \\ Correspondence should be addressed to G. Elsalamony, elsalamony@hotmail.com
}

Received 17 February 2009; Accepted 25 May 2009

Recommended by Etienne Kerre

The present paper investigates the relations between fuzzifying topologies and generalized ideals of fuzzy subsets, as well as constructing generalized ideals and fuzzifying topologies by means of fuzzy preorders. Furthermore, a construction of generalized ideals from preideals, and vice versa, is obtained. As a particular consequence of the results in this paper, a construction of fuzzifying topology generated by generalized ideals of fuzzy subsets via a given $I$-topology is given. The notion of $\sigma$-generalized ideal is introduced and hence every $\sigma$-generalized ideal is shown to be a fuzzifying topology induced by some fuzzy preorder.

Copyright (C) 2009 G. Elsalamony. This is an open access article distributed under the Creative Commons Attribution License, which permits unrestricted use, distribution, and reproduction in any medium, provided the original work is properly cited.

\section{Introduction}

The concepts of preideals and generalized ideals were introduced by Ramadan et al. [1], as a consistent approach to the ideas of fuzzy mathematics. The authors investigated the interrelations between these two concepts. This paper is devoted to finding fuzzifying topologies derived from preideals, and vise versa, by means of fuzzy preorders and residual implications. The contents of this paper are arranged as follows. In Section 2, we recall some basic notions of ideals, preideals, and generalized ideals. The notion of saturation of preideals is introduced, hence, a one-to-one correspondence between the set of saturated preideals and the set of generalized ideals on the same set is obtained. In fact, some studies were done on the correspondence of such two sets, see [2,3], for example, based on the duality between fuzzy filters and fuzzy ideals. In our paper, we prove such correspondence independent of the duality principle. Also, given $X$ a Boolean lattice, we construct a generalized ideal, in terms of fuzzy preorders. Section 3 is concerned with the relationship between the fuzzy preorders and each of $I$-topologies [4], fuzzifying topologies [5], and $I$-fuzzy topologies [6], respectively. Thus, an interesting relation between a special type of $I$-topological spaces, 
called fuzzy neighborhood spaces, introduced by Lowen [7], and fuzzifying topological spaces is established. In Section 4, we introduce the notion of $\sigma$-generalized ideal, therefore, a characterization of this special kind of ideals is shown to be a fuzzifying topology generated by some fuzzy preorder. Also, we study the relations between preideals and generalized topological structures, for example, I-topologies, fuzzifying topologies, and Ifuzzy topologies. Also, I-topologies are constructed from preideals via given $I$-topologies.

\section{Generalized Ideal Structures}

We recall some basic definitions. We should let $X, I$ denote a nonempty set and the closed unit interval $[0,1]$, respectively, and we let $I_{0}=(0,1], I_{1}=[0,1)$.

We denote the characteristic function of a subset $A \subseteq X$ also by $A$. If $\mu \in 1^{X}$, we define $\mu^{\alpha}=\{x \in X: \mu(x)>\alpha\}$. Let $2^{X}$ be the collection of all subsets of $X$. The fuzzy set which assigns to each element in $X$ the value $\alpha, 0 \leq \alpha \leq 1$, is also denoted by $\alpha$. In this paper, the concepts of triangular norm and residual implication are applied.

A triangular norm, a $t$-norm in short, on the unit interval $I$ is a binary operator $*$ : $I \times I \rightarrow I$ which is symmetric, associative, order-preserving on each place and has 1 as the unit element.

A fuzzy relation on a nonempty set is a map $R: X \times X \rightarrow I$. A fuzzy relation $R$ is called

(1) reflexive if $R(x, x)=1$ for each $x \in X$;

(2) $*$-transitive if $R(x, y) * R(y, z) \leq R(x, z)$ for all $x, y, z \in X$.

A reflexive and $*$-transitive fuzzy relation is called $*$-fuzzy preorder. If $*$ is assumed to be the $t$-norm $\min =\wedge$, we will drop the $*$-.

This section presents a review of some fundamental notions of ideals, preideals, and generalized ideals. We refer to Ramadan et al. [1] for details.

Definition 2.1. A nonempty subset $D$ of $2^{X}$ is an ideal of subsets of $X$, simply, an ideal on $X$, if it satisfies the following conditions:

(D1) $X \notin D$;

(D2) $A, B \in D \Rightarrow A \cup B \in D$;

(D3) $A \in D, B \subset A \Rightarrow B \in D$.

Definition 2.2. A nonempty subset $\Phi$ of $I^{X}$ is a preideal of subsets of $X$, simply, a preideal on $X$, if it satisfies the following conditions:

(P1) $1 \notin \Phi$;

(P2) $\mu, v \in \Phi \Rightarrow \mu \vee v \in \Phi$;

(P3) $v \in \Phi, \mu \leq v \Rightarrow \mu \in \Phi$.

A preidael $\Phi$ is called prime if it satisfies the condition;

$$
\forall \mu, \lambda \in I^{X} \text { such that } \mu \wedge v \in \boldsymbol{\Phi} \text {, then } \mu \in \boldsymbol{\Phi} \text { or } v \in \boldsymbol{\Phi} \text {. }
$$

A preideal $\Phi$ is called a $\sigma$-preideal if the following hold. 
If $\left\{\mu_{n}: n=1,2,3, \ldots\right\}$ is a countable subcollection of $\boldsymbol{\Phi}$, then $\sup _{n} \mu_{n} \in \boldsymbol{\Phi}$.

If $\Phi$ is a preideal on $X$, we define the characteristic, denoted by $c(\Phi)$, of $\Phi$ by

$$
c(\Phi)=\sup _{\mu \in \Phi} \inf \mu(x)
$$

If $\Phi$ is a preideal and $c(\Phi)<1$, we say that $\Phi$ is saturated if for every $\left(\mu_{\varepsilon}\right) \in \Phi^{I_{1}}$, $\inf _{\varepsilon \in I_{1}}\left(\mu_{\varepsilon}+\varepsilon\right) \in \Phi$, equivalently, $\Phi$ is saturated if it satisfies

$$
\forall \varepsilon<1, \quad \mu \in I^{X}: \mu-\varepsilon \in \Phi \Longrightarrow \mu \in \Phi \text {. }
$$

We provide a useful characterization of a saturated preideal $\Phi$ in the following lemma.

Lemma 2.3. Let $\boxplus$ be a saturated preideal on $X, \mu \in I^{X}$. Then

$$
\mu \in \Phi \Longleftrightarrow \mu^{\alpha} \wedge \gamma \in \Phi, \quad \forall c<\gamma<\alpha \text { in } I_{1}
$$

Proof. Let $\mu \in \Phi$. Since $\mu^{\alpha} \wedge \gamma \leq \mu$ for all $\gamma<\alpha \in I_{1}$, then, by (P3), $\mu^{\alpha} \wedge \gamma \in \Phi$ for all $\gamma<\alpha \in I_{1}$. Conversely, let $\mu \in I^{X}$ such that $\mu^{\alpha} \wedge \gamma \in \Phi$ for all $c<\gamma<\alpha$ in $I_{1}$. For every $\alpha \in(0,1 / 2)$ and $0<n$ (integer) $<1 / \alpha$, we consider the fuzzy subsets $\mu_{2 \alpha}=\sup _{n}\left(\mu^{n \alpha} \wedge(n \alpha-\alpha)\right)$. Since $n$ is finite, then, by (P2), $\mu_{2 \alpha} \in \mathcal{D}$. Also, it is easy to see that, for all $x \in X, 0 \leq \mu(x)-\mu_{2 \alpha}(x) \leq 2 \alpha$, hence $\mu+2 \alpha \geq \mu_{2 \alpha}+2 \alpha \geq \mu$. Consequently, $\mu=\cap_{0<\alpha<1 / 2}\left(\mu_{2 \alpha}+2 \alpha\right) \in \Phi$, since $\Phi$ is saturated, so we are done.

It should be noticed that the required condition of Lemma 2.3 holds, trivially, for all $\mu \in \Phi$ and $\gamma \leq c$, therefore, we provide $\gamma>c$, so that the above condition is significant.

Also, the characteristic of a preideal $\Phi$, on a subset $A$ of $X$ may be defined and denoted by $c_{A}(\boldsymbol{\Phi})=\sup _{\lambda \in \Phi} \inf _{x \in A} \lambda(x)$. The following result is useful for our study.

Lemma 2.4. Let $\Phi$ on $X$. Then, for every $A \in 2^{X}$,

$$
c_{A}(\boldsymbol{\Phi})=\sup \{t \in I: A \wedge t \in \Phi\}
$$

hence, $c=c_{X}(\Phi)=\sup \{t \in I: t \in \Phi\}$.

Proof. The statement holds when $A=\phi$. Suppose $A \neq \phi$, and denote $c=c_{A}(\boldsymbol{\Phi})$ and $b=$ $\sup \{t \in I: A \wedge t \in \boldsymbol{\Phi}\}$. Let $t \in I$ satisfy $A \wedge t \in \boldsymbol{\Phi}$. Then $c \geq \inf _{x \in A}(A \wedge t)(x)=t$, that is, $c \geq b$. Conversely, given $\lambda \in \Phi$, let $\gamma=\inf _{x \in A} \lambda(x)$, hence $A \wedge \gamma \leq \lambda$. This implies, by (P3), $A \wedge \gamma \in \Phi$, so $b \geq \gamma$. Thus, by definition of $c$, we get $b \geq c$, this shows that the equality holds. 
Definition 2.5. A nonzero function $d: 2^{X} \rightarrow I$ is called a generalized ideal of fuzzy subsets of $X$, simply, a generalized ideal on $X$, if it satisfies the following conditions:

(G1) $d(X)=0$;

(G2) $d(A) \wedge d(B) \leq d(A \cup B)$, for each $A, B \in 2^{X}$;

(G3) if $A \subset B$, then $d(A) \geq d(B)$, for each $A, B \in 2^{X}$;

The conditions (G2) and (G3) are equivalent to:

$\left(\mathrm{G}^{*}\right) d(A) \wedge d(B)=d(A \cup B)$, for each $A, B \in 2^{X}$. Moreover, Since $d$ a nonzero function, then by $(\mathrm{G} 3), d(\phi)>0$.

A generalized ideal $d$, is called $\sigma$-generalized ideal if

$$
d\left(\cup_{i \in J} A_{i}\right) \geq \underset{i \in J}{\wedge} d\left(A_{i}\right), \quad \text { for each }\left(A_{i}\right)_{i \in J} \subseteq I^{X},
$$

and $d$ is called prime if

$$
d(A \cap B) \leq d(A) \vee d(B), \quad \text { for each } A, B \in 2^{X} .
$$

A generalized ideal on a set $X$ can be derived by fuzzy relations in different ways, as we see in the following.

Proposition 2.6. Let $R$ be a reflexive fuzzy relation on a Boolean lattice $X$ with 0 . Define $d_{1}, d_{2}$ : $I^{X} \rightarrow I$, for all $A \subseteq X$ as follows:

$$
\begin{gathered}
d_{1}(A)=\underset{x, y \in A}{\wedge}(1-R(x, 1-y)) ; \quad \text { where } 1-y \text { is the complement } y \\
d_{2}(A)=\underset{x \in A}{\wedge}(1-R(x, 0)) .
\end{gathered}
$$

Then $d_{1}, d_{2}$ are generalized ideal on $X$.

Proof. Straightforward.

Now, we will construct different types of preideals derived from a generalized ideals.

Proposition 2.7. Let $d$ be a generalized ideal on X. Define $\Phi_{1}, \Phi_{2} \subset I^{X}$ by

$$
\begin{gathered}
\Phi_{1}=\left\{\mu \in I^{X}: \forall A \in 2^{X}, d(A) \leq 1-\sup _{x \notin A} \mu(x)\right\} ; \\
\Phi_{2}=\left\{\mu \in I^{X}: \forall \alpha \in I_{1}, d\left(\mu^{\alpha}\right) \geq \alpha\right\} .
\end{gathered}
$$

Then both $\Phi_{1}, \Phi_{2}$ are saturated preideals on $\mathrm{X}$. 
Proof. First, we show that $\Phi_{1}$ a preideal on $X$ :

(P1) $1 \notin \Phi_{1}$, otherwise for all $A \in 2^{X}, d(A) \leq 1-\sup _{x \notin A} 1(x)=0$, that is, $d(A)=0$, which is a contradiction, since $d$ is a nonzero function;

(P2) let $\mu, \lambda \in \Phi_{1}$. Then for all $A \in 2^{X}$ :

$$
1-\sup _{x \notin A}(\mu \vee \lambda)(x)=\left(1-\sup _{x \notin A} \mu(x)\right) \wedge\left(1-\sup _{x \notin A} \lambda(x)\right) \geq d(A) \wedge d(A)=d(A),
$$

hence $\mu \vee \lambda \in \Phi_{1}$.

(P3) Let $\mu, \lambda \in I^{X}$ such that $\mu \in \Phi_{1}$ and $\lambda \leq \mu$. Then for all $A \in 2^{X}$,

$$
1-\sup _{x \notin A} \lambda(x) \geq 1-\sup _{x \notin A} \mu(x) \geq d(A), \quad \text { hence } \lambda \in \Phi_{1} \text {. }
$$

Let $\left(\mu_{\theta}\right)_{\theta \in I_{0}}$ be a family of fuzzy sets in $\Phi_{1}$. Then for all $A \in 2^{X}$,

$$
\begin{aligned}
1-\sup _{x \notin A}\left(\inf \left(\mu_{\theta}+\theta\right)\right)(x) & =1-\sup _{x \notin A}\left(\inf _{\theta \in I_{0}}\left(\mu_{\theta}(x)+\theta\right)\right) \\
& \geq 1-\inf _{\theta \in I_{0}} \sup _{x \notin A}\left(\mu_{\theta}(x)+\theta\right) \\
& =\sup _{\theta \in I_{0}}\left(1-\sup _{x \notin A}\left(\mu_{\theta}(x)+\theta\right)\right) \\
& =\sup _{\theta \in I_{0}}\left(1-\left(\sup _{x \notin A} \mu_{\theta}(x)+\theta\right)\right) \\
& =\sup _{\theta \in I_{0}}\left(\left(1-\sup _{x \notin A} \mu_{\theta}(x)\right)-\theta\right) \\
& \geq \sup _{\theta \in I_{0}}(d(A)-\theta)=d(A),
\end{aligned}
$$

therefore, $\inf _{\theta \in I_{0}}\left(\mu_{\theta}+\theta\right) \in \boldsymbol{\Phi}_{1}$, hence $\boldsymbol{\Phi}_{1}$ is saturated.

Second, we prove that $\Phi_{2}$ is saturated preideal on $X$ :

(P1) $1 \notin \Phi_{2}$, otherwise $d\left(1^{\alpha}\right)=d(X)=0 \nsupseteq \alpha$, for all $\alpha \in I_{1}$;

(P2), (P3) direct.

Let $\left(\mu_{\theta}\right)_{\theta \in I_{0}}$ be a family of fuzzy sets in $\Phi_{2}$. Then

$$
\begin{aligned}
d\left(\left(\inf _{\theta \in I_{0}}\left(\mu_{\theta}+\theta\right)\right)^{\alpha}\right) & \geq d\left(\bigcap_{\theta \in I_{0}}\left(\mu_{\theta}+\theta\right)^{\alpha}\right) \\
& \geq \sup _{\theta \in I_{0}} d\left(\mu_{\theta}^{\alpha-\theta}\right), \quad(\text { by }(\mathrm{G} 3)) \geq \sup _{\theta \in I_{0}}(\alpha-\theta)=\alpha .
\end{aligned}
$$

Hence $\Phi_{2}$ is saturated preideal. 
Proposition 2.8. Let $d$ be a prime generalized ideal on $X$. Define $\Phi_{3}, \Phi_{4} \subset I^{X}$ by

$$
\begin{aligned}
& \Phi_{3}=\left\{\mu \in I^{X}: \forall \alpha \in I_{1}, d\left((1-\mu)^{\alpha}\right) \leq \alpha\right\} ; \\
& \Phi_{4}=\left\{\mu \in I^{X}: \forall A \in 2^{X}, d(A) \leq \sup _{x \notin A}(1-\mu)(x)\right\} .
\end{aligned}
$$

Then $\Phi_{3}, \Phi_{4}$ are prime saturated preideals on X. Moreover $\Phi_{3}=\Phi_{4}$.

Proof. First, we show that $\Phi_{3}=\Phi_{4}$. Let $\mu \in I^{X}$ such that $d\left((1-\mu)^{\alpha}\right) \leq \alpha$, for all $\alpha \in I_{1}$. If $A \in 2^{X}$, we assume that $\sup _{x \notin A}(1-\mu)(x)=\gamma \in I_{1}$, hence $(1-\mu)^{\gamma} \subseteq A$.

Then, by $(\mathrm{G} 3), d(A) \leq d\left((1-\mu)^{\gamma}\right) \leq \gamma=\sup _{x \notin A}(1-\mu)(x)$, that implies $\Phi_{3} \subseteq \Phi_{4}$.

Conversely, let $\mu \in I^{X}$ such that $d(A) \leq \sup _{x \notin A}(1-\mu)(x)$, for all $A \in 2^{X}$. Then $d\left((1-\mu)^{\alpha}\right) \leq \sup _{x \notin(1-\mu)^{\alpha}}(1-\mu)(x) \leq \alpha$, for all $\alpha \in I_{1}$ this implies $\boldsymbol{\Phi}_{4} \subseteq \Phi_{3}$, thus $\Phi_{3}=\Phi_{4}$.

Second, we show that $\Phi_{3}$ is a saturated prime preideal.

(P1) Suppose that $1 \in \Phi_{3}$. Let $d(\phi)=\gamma>0$. Then, for all $\alpha<\gamma, d\left(0^{\alpha}\right)=d(\phi)=\gamma>\alpha$, which is a contradiction, so $1 \notin \Phi_{3}$.

(P2) Let $\mu, \lambda \in \Phi_{3}$. Then for all $\alpha \in I_{1}, d\left(1-(\mu \vee \lambda)^{\alpha}\right)=d\left((1-\mu)^{\alpha} \cap(1-\lambda)^{\alpha}\right) \leq$ $d\left((1-\mu)^{\alpha}\right) \vee d\left((1-\lambda)^{\alpha}\right) \leq \alpha$, since $d$ is prime. This implies $\mu \vee \lambda \in \Phi_{3}$.

(P3) Let $\mu, \lambda \in I^{X}$ such that $\mu \in \Phi_{3}$ and $\lambda \leq \mu$. Then $d\left((1-\lambda)^{\alpha}\right) \leq d\left((1-\mu)^{\alpha}\right) \leq \alpha$, by (G3), hence $\lambda \in \Phi_{3}$.

Now, we will show that $\Phi_{4}$ is saturated. Suppose for all $\varepsilon>0, \mu-\varepsilon \in D_{3}$. Then for all $A \in 2^{X}, d(A) \leq \sup _{x \notin A}(1-(\mu-\varepsilon))(x)=\sup _{x \notin A}(1-\mu)(x)+\varepsilon$, hence $d(A) \leq \sup _{x \notin A}(1-\mu)(x)$, that is, $\Phi_{4}$ is saturated.

Finally, we show that $\Phi_{3}$ is prime. Let $\mu, \lambda \in I^{X}$ such that $\mu \wedge \lambda \in \Phi_{3}$. Then for all $\alpha \in I_{1}, d\left(1-\mu^{\alpha}\right) \wedge d\left(1-\lambda^{\alpha}\right) \leq d\left(\left(1-\mu^{\alpha}\right) \cup\left(1-\lambda^{\alpha}\right)\right)=d\left(1-\left(\mu^{\alpha} \wedge \lambda^{\alpha}\right)\right)=d\left(1-(\mu \wedge \lambda)^{\alpha}\right) \leq \alpha$, hence, $d\left(1-\mu^{\alpha}\right) \leq \alpha$ or $d\left(1-\lambda^{\alpha}\right) \leq \alpha$, that implies $\mu \in \Phi_{3}$ or $\lambda \in \Phi_{3}$.

Proposition 2.9. Let $\Phi$ be a preideal on X. Define $d: 2^{X} \rightarrow I$ by

$$
\forall A \in 2^{X}, \quad d(A)=\sup \{t \in I: t>c, A \wedge t \in \Phi\} .
$$

Then $d$ is a generalized ideal on $X$.

Proof. (G1) $d(X)=0$, since $t \in \Phi$ implies $t \leq c$.

(G2) Let $A, B \in 2^{X}$ and $\alpha, \beta \in I, \alpha>c, \beta>c$ such that $A \wedge \alpha \in \Phi, B \wedge \beta \in \Phi$.

Then, by $(\mathrm{P} 2),(A \wedge \alpha) \vee(B \wedge \beta) \in \mathcal{D}$. Let $\gamma=\min (\alpha, \beta)$. Then, by (P3), we get $(A \cup B) \wedge \gamma=$ $(A \wedge \gamma) \vee(B \wedge \gamma) \in \mathbb{D}$, and $\gamma=\alpha \wedge \beta>c$. Thus $\sup \{t: t>c,(A \cup B) \wedge t \in \mathbb{Q}\} \geq \gamma=\alpha \wedge \beta$, hence $\sup \{t: t>c,(\mathrm{~A} \cup \mathrm{B}) \wedge t \in \mathbb{\Phi}\} \geq \sup \{t: t>c, A \wedge t \in \boldsymbol{\Phi}\} \wedge \sup \{t: t>c, B \wedge t \in \mathbb{\Phi}\}$, so $d(A \cup B) \geq d(A) \wedge d(B)$.

Now we come to the main result of this section.

Theorem 2.10. There is a one-to-one correspondence between the set saturated preideals on a nonempty set $X$ and the set of generalized ideals on $X$. 
Proof. Let $d$ be a generalized ideal on X. Denote the preideal $\Phi_{2}$ generated by $d$, defined as in Proposition 2.7, by $\Phi_{d}$, and denote the generalized ideal, generated by $\Phi_{d}$, as in Proposition 2.9, by $d_{\Phi_{d}}$, hence for all $A \in 2^{X}$

$$
\begin{aligned}
d_{\Phi_{d}}(A) & =\sup \left\{t \in I_{1}: t>c, A \wedge t \in \Phi_{d}\right\}, \quad \text { Proposition } 2.9 \\
& =\sup \left\{t \in I_{1}: t>c, d\left((A \wedge t)^{\alpha}\right) \geq \alpha, \forall \alpha \in I_{1}\right\}, \quad \text { (2.11) in Proposition } 2.7 \\
& =\sup \left\{t \in I_{1}: t>c, d(A) \geq \alpha, \forall \alpha<t\right\}, \quad \text { since } d(\phi) \geq d(A), \forall A \subseteq X \\
& =\sup \left\{t \in I_{1}: t>c, d(A) \geq t\right\} \\
& =d(A) .
\end{aligned}
$$

Conversely, let $\Phi$ be a saturated preideal on $X$. Denote the generalized ideal generated by $\Phi$, as in Proposition 2.9, by $d_{\Phi}$, and denote the preideal generated by $d_{\Phi}$, as in (2.11) in Proposition 2.7 by $\Phi_{d_{\Phi}}$. Then,

$$
\begin{aligned}
\Phi_{d_{\Phi}} & =\left\{\lambda \in I^{X}: d_{\Phi}\left(\lambda^{\alpha}\right) \geq \alpha, \forall \alpha \in I_{1}\right\} \\
& =\left\{\lambda \in I^{X}: \sup \left\{t \in I_{1}, t>c, \lambda^{\alpha} \wedge t \in \Phi\right\} \geq \alpha, \forall \alpha \in I_{1}\right\} \\
& =\left\{\lambda \in I^{X}: \lambda^{\alpha} \wedge t \in \Phi, \forall c<t<\alpha\right\} \\
& =\left\{\lambda \in I^{X}: \lambda \in \Phi\right\}, \quad \text { by Lemma } 2.3 \\
& =\Phi .
\end{aligned}
$$

This completes the proof.

Definition 2.11. A nonzero function $\mathfrak{d}: I^{X} \rightarrow I$ is called a fuzzy $I$-ideal on $X$ if it satisfies the following conditions:

(d1) $\mathfrak{d}(1)=0$;

(d2) $\mathfrak{d}(\lambda \vee \mu) \geq \mathfrak{d}(\lambda) \wedge \mathfrak{d}(\mu)$, for $\lambda, \mu \in I^{X}$;

(d3) if $\lambda \geq \mu$, then $\mathfrak{d}(\mu) \geq \mathfrak{d}(\lambda)$.

Clearly, a generalized ideal $d$ on a set $X$ can be regarded as a fuzzy $I$-ideal whose domain is $2^{X}$. Precisely, $d(A)=\mathfrak{d}(A)$ for each $A \subseteq X$; otherwise $d(A)=0$.

Remark 2.12 (Fang and Chen [8]). Defined a generalized fuzzy preorder $R_{\phi}$, corresponding to a $\operatorname{map} \phi: I^{X} \rightarrow I$ as follows:

$$
\forall(x, y) \in X \times X, \quad R_{\phi}(x, y)=\underset{\mu \in I^{X}}{\wedge} \phi(\mu) \longrightarrow(\mu(x) \rightarrow \mu(y))
$$

hence, for a fuzzy $I$-ideal $\mathfrak{d}$ on $X$, there corresponds a fuzzy preorder $R_{\mathfrak{d}}$ defined by

$$
\forall(x, y) \in X \times X, \quad R_{\mathfrak{d}}(x, y)=\underset{\mu \in I^{X}}{\wedge} \mathfrak{d}(\mu) \longrightarrow(\mu(x) \rightarrow \mu(y)) .
$$


Especially, for a generalized ideal $d$ on $X$, the corresponding fuzzy preorder of $d$ is given by the following: For every $(x, y) \in X \times X$,

$$
R_{d}(x, y)=\bigwedge_{\substack{A \subseteq X \\(x, y) \in A^{\prime} \times A}}(d(A) \rightarrow 0)
$$

\section{Connecting Fuzzy Preorders and $I$-fuzzy Topologies}

In this section, we recall some basic results about the connection between fuzzy preorders and each of $I$-topologies, fuzzifying topologies, and I-fuzzy topologies. Also, the relations among these different types of topologies are investigated.

An $I$-topology on a set $X$ is a crisp subset $\tau$ of $I^{X}$ which is closed with respect to finite meets and arbitrary joins and which contains all the constant functions from $X$ to $I$. If the constants are 0,1 only, we will call it a Chang I-topology.

Another more consistent approach to the fuzziness has been developed. According to Shostak [6], an $I$-fuzzy topology on a set $X$ is a map $\mathfrak{I}: I^{X} \rightarrow I$, satisfying the following conditions:

(1) $\Im(\alpha)=1$ for all constant functions $\alpha: X \rightarrow I$;

(2) $\mathfrak{I}(\mu \wedge \lambda) \geq \Im(\mu) \wedge \Im(\lambda)$, for all $\mu, \lambda \in I^{X}$;

(3) $\Im\left(\vee_{j \in J} \mu_{i}\right) \geq \wedge_{j \in J} \Im\left(\mu_{j}\right), j \in J, \mu_{j} \in I^{X}$.

By the conditions (1) and (2), we get for $\mu \in I^{X}$ and $\alpha \in I_{0}, \Im(\mu \wedge \alpha) \geq \Im(\mu)$. An $I$-fuzzy topology on a set $X$ is called trivial, if $\Im(\lambda)=1$, for all $\lambda \in I^{X}$, and is called homogeneous if the following condition holds: for $\mu \in I^{X}$ and $\alpha \in I, \Im(\mu \wedge \alpha)=\Im(\mu)$.

If a map $\tau: 2^{X} \rightarrow I$ satisfies the similar conditions of $I$-fuzzy topology on a set $X$, then $\tau$ is called a fuzzifying topology Ying [9]. Furthermore, if a fuzzifying topology $\tau$ satisfies the following condition: topology.

(4) for all $\left\{A_{j}\right\}_{j \in J} \subseteq 2^{X}, \tau\left(\cap_{j \in J} A_{j}\right) \geq \wedge_{j \in J} \tau\left(A_{j}\right)$, then $\tau$ is called a saturated fuzzifying

For a given left-continuous $t$-norm $*: I \times I \rightarrow I$, the corresponding residual implication $\rightarrow: I \times I \rightarrow I$ is given by $x \rightarrow y=\sup \{z \in I: x * z \leq y\}$.

Many properties of residual implications are found in litrature, but we will recall some of them, which will be used in this paper:

$$
\begin{aligned}
& \text { (I1) } x \rightarrow y=1 \text { if and only if } x \rightarrow \mathrm{y} ; \\
& \text { (I2) } 1 \rightarrow x=x \text {; } \\
& \text { (I3) }(x \rightarrow y) *(y \rightarrow z) \leq x \rightarrow z ; \\
& \text { (I4) } x \rightarrow \wedge_{j \in J} y_{j}=\wedge_{j \in J}\left(x \rightarrow y_{j}\right) \text {, hence, } x \rightarrow y \leq x \rightarrow z \text { if } y \leq z ; \\
& \text { (I5) }\left(\vee_{j \in J} y_{j}\right) \rightarrow y=\wedge_{j \in J}\left(y_{j} \rightarrow y\right) \text {, hence } x \rightarrow z \geq y \rightarrow z \text { if } x \leq y \text {; } \\
& \text { (I6) } x * \vee_{j \in J} y_{j}=\vee_{j \in J}\left(x * y_{j}\right) \text {; } \\
& \text { (I7) } x \rightarrow(y \rightarrow z)=y \rightarrow(x \rightarrow z) \text {; } \\
& \text { (I8) }(x \rightarrow y) *(u \rightarrow v) \leq x * u \rightarrow y * v ; \\
& \text { (I9) } x \leq(x \rightarrow y) \rightarrow y \text {; } \\
& \text { (I10) } x \rightarrow y=\wedge_{\curlywedge \in I}((\lambda \rightarrow x) \rightarrow(\lambda \rightarrow y))
\end{aligned}
$$




$$
\begin{aligned}
& \text { (I11) } x \rightarrow y=\wedge_{\curlywedge \in I}((y \rightarrow \lambda) \rightarrow(x \rightarrow \lambda)) \\
& \text { (I12) }(x \wedge y) \rightarrow(z \wedge w) \geq(x \rightarrow z) \wedge(y \rightarrow w) .
\end{aligned}
$$

Clearly, by (I1) and (I3), the residual implication $\rightarrow$ with respect to a $t$-norm $*$ is a fuzzy preorder on $I$ and called the canonical fuzzy preorder on $I$.

The relationships between fuzzy preorders and I-topologies, fuzzifying topologies, and I-fuzzy topologies, respectively, have been investigated by many authors. For example, we recall the main results of Lai and Zhang [10], Fang and Chen [8], and Fang [11].

Theorem 3.1 (Lai and Zhang [10]). For a fuzzy preorder $R$ on a set $X$, the family $\tau_{R}=\{\mu \in$ $I^{X}: R(x, y) * \mu(x) \leq \mu(y)$, for all $\left.x, y \in X\right\}$, is an I-Alexandrov topology induced by $R$, that is, $\tau_{R}$ satisfies the folowing properties: for all $F \subseteq \tau_{R}, \mu \in \tau_{R}$, and $\alpha \in I$,

(a) every constant fuzzy set $X \rightarrow I$ belongs to $\tau_{R}$;

(b) $\vee \mathcal{F} \in \tau_{R}$;

(c) $\wedge \mathcal{F} \in \tau_{R} ;$

(d) $\alpha * \mu \in \tau_{R}$;

(e) $\alpha \rightarrow \mu \in \tau_{R}$.

Moreover, $R$ satisfies the equality

$$
R(x, y)=\bigwedge_{\mu \in \tau_{R}} \mu(x) \longrightarrow(y) .
$$

Conversely, for a given I-Alexandrov topology $\tau$ a set $X$, there is a unique $*$-fuzzy preorder $R_{\tau}$, given by $R_{\tau}(x, y)=\Lambda_{\mu \in \tau} \mu(x) \rightarrow \mu(y)$, such that $\tau=\tau_{R_{\tau}}$.

Thus, there is a one-to-one correspondence between the set of I-Alexandrov topologies on a set $X$ and $*$-fuzzy preorders.

Theorem 3.2 (Fang and Chen [8]). Every fuzzy preorder $R$ on a set $X$ corresponds a saturated fuzzifying topology $\tau_{R}$ on $X$, given by for all $A \subseteq X$,

$$
\tau_{R}(A)={\hat{(x, y) \in A \times A^{\prime}}}_{(1-R(x, y))}(1
$$

conversely, every fuzzifying topology $\tau$ on a set $X$ corresponds a fuzzy preorder $R_{\tau}$, given by: for all $x, y \in X$,

$$
R_{\tau}(x, y)={\hat{(x, y) \in A \times A^{\prime}}}(1-\tau(A))
$$

Thus, there is a one-to-one correspondence between the set of saturated fuzzifying topologies on a set $X$ and the set of fuzzy preorders on $X$.

By the above two theorems, one can dedeuce, at once, the following corollary.

Corollary 3.3. The set of saturated fuzzifying toplogies on a set $X$ and the set of I-Alexandrov topologies on $\mathrm{X}$ are in one-to-one correspondence. 
A special type of I-topological spaces, called fuzzy neighborhood spaces, was introduced by Lowen [7]. The interrelations between these spaces and the fuzzifying toplogical spaces are interesting to study. Although a study of fuzzifying topological spaces and their relation with fuzzy neighborhood spaces has been investigated, sestematically, see, for example, [12], we give, in this section, alternative methods. We recall the folowing definition.

Definition 3.4 (Wuyts et al. [13]). An I-topological space $(X, \tau)$ is called a fuzzy neighborhood space if whenever $\mu \in \tau$, for any $\alpha \in I_{1}$ also $\alpha \wedge \mu^{\alpha} \in \tau$.

Equivalently, if whenever $\mu \in \tau$, for any $\alpha, t \in I_{1}, t<\alpha$ also $\mu^{\alpha} \wedge t \in \tau$.

Proposition 3.5. Let $\tau$ be a fuzzifying topology on a set X. Then $\tau$ corresponds an I-topology $\tau_{\tau}$ on $X$, defined by $\tau_{\tau}=\left\{\mu \in I^{X}: \tau\left(\mu^{\alpha}\right) \geq \alpha\right.$, for all $\left.\alpha \in I_{1}\right\}$, and $\left(X, \tau_{\tau}\right)$ is a fuzzy neighborhood space.

Conversely, let $\tau$ be an I-topology on $X$. Then $\tau$ corresponds a fuzzifying topology $\tau \tau$, on $X$, defined by: for all $A \subseteq X, \tau \tau(A)=\sup \{\alpha \in I: A \wedge \alpha \in \tau\}$. Moreover,

(1) $\tau \tau_{\tau}=\tau$,

(2) $\tau_{\tau \tau} \leq \tau$ and if $(X, \tau)$ is a fuzzy neighborhood space, then $\tau_{\tau \tau}=\tau$.

Proof. That is, $\tau_{\tau}$ and $\tau \tau$ are $I$-topology and fuzzifying topology on $X$, respectively, and are easy to be verified. To prove that $\left(X, \tau_{\tau}\right)$ is a fuzzy neighborhood space, we suppose $\alpha, t \in I$ and $\mu \in \tau_{\tau}$, then

$$
\tau\left(\left(\mu^{t} \wedge t\right)^{\alpha}\right)= \begin{cases}\tau(\phi)=1, & \text { if } \alpha \geq t \\ \tau\left(\mu^{t}\right) \geq t, & \text { if } t>\alpha .\end{cases}
$$

Therefore, $\tau\left(\left(\mu^{t} \wedge t\right)^{\alpha}\right) \geq \alpha$, for all $\alpha \in I_{1}$, that is, $\mu^{t} \wedge t \in \tau_{\tau}$, for all $t \in I_{1}$, so we are done.

To prove (1), let $\tau$ be a fuzzifying topology on $X$ then for all $\wedge \subseteq X$,

$$
\begin{aligned}
\tau_{\tau_{\tau}}(A) & =\sup \left\{\alpha \in I: A \wedge \alpha \in \tau_{\tau}\right\} \\
& =\sup \left\{\alpha \in I: \tau\left((A \wedge \alpha)^{t}\right) \geq t, \forall t \in I_{1}\right\} \\
& =\sup \{\alpha \in I: \tau(A) \geq t, \forall t<\alpha\}, \quad \text { since } \tau(\phi)=1 \\
& =\tau(A) .
\end{aligned}
$$

To prove (2), suppose that $(X, \tau)$ is a fuzzy neighborhood space. Then

$$
\begin{aligned}
\tau_{\tau \tau} & =\left\{\mu \in I^{X}: \tau_{\tau}\left(\mu^{\alpha}\right) \geq \alpha, \forall \alpha \in I_{1}\right\} \\
& =\left\{\mu \in I^{X}: \sup \left\{t \in I: \mu^{\alpha} \wedge t \in \tau\right\} \geq \alpha, \forall \alpha \in I_{1}\right\} \\
& =\left\{\mu \in I^{X}: \mu^{\alpha} \wedge t \in \tau, \forall \mathrm{t}<\alpha, \alpha \in I_{1}\right\} \leq \tau, \\
& =\left\{\mu \in I^{X}: \mu \in \tau\right\}=\tau, \quad \text { if } \tau \text { is a fuzzy neighborhood structure. }
\end{aligned}
$$


Therefore, as a direct result of Proposition 3.5, we have the following.

Theorem 3.6. There is a one-to-one correspondence between the set of fuzzifying topologies and the set of fuzzy neighborhood structures on the same set.

Definition 3.7. Given $(X, \mathfrak{I})$, an $I$-fuzzy topological space, and $\alpha \in I_{0}$, then $\Im_{\alpha}:=\left\{\mu \in I^{X}\right.$ : $\Im(\mu) \geq \alpha\}$ is an $I$-topology on $X$, called the $\alpha$-level $I$-topology of $\mathfrak{I}$.

Consequently, $\tau_{\mathfrak{I}}:=\mathfrak{I}_{1}=\left\{\mu \in I^{X}: \mathfrak{I}(\mu)=1\right\}$, by which we will give an interrelation between $I$-topologies and $I$-fuzzy topologies.

Proposition 3.8. Let $\tau$ be an I-topology on $X$. Then for all $\mu \in I^{X}, \Im_{\tau}(\mu):=\sup \left\{\alpha \in I_{1}: \mu \wedge \alpha \in \tau\right\}$ is a homogeneous I-fuzzy topology on X.

Proof. That is, $\Im_{\tau}$ an $I$-fuzzy topology is easy to be verified. Now, let $\mu \in I^{X}, \alpha \in I$, then we get

$$
\begin{aligned}
\Im_{\tau}(\mu \wedge \alpha) & :=\sup \left\{t \in I_{1}: \mu \wedge \alpha \wedge t \in \tau\right\} \\
& \leq \sup \left\{t \in I_{1}: t \leq \alpha, \mu \wedge \alpha \wedge t \in \tau\right\} \\
& =\sup \left\{t \in I_{1}: \mu \wedge t \in \tau\right\} \\
& =I_{\tau}(\mu) .
\end{aligned}
$$

Since $\mathfrak{I}_{\tau}$ is an $I$-fuzzy topology, then $\mathfrak{I}_{\tau}(\mu \wedge \alpha) \geq \Im(\mu)$, so the equality holds.

Theorem 3.9. There is a one-to-one correspondence between the set of I-topologies on a set X and the set of homogeneous I-fuzzy topologies on X.

Proof. Let $\tau$ be an $I$-topology on $X$, then $\Im_{\tau}$ is a homogeneous $I$-fuzzy topology on $X$, by Proposition 3.8, and

$$
\begin{aligned}
\tau_{\mathfrak{I}_{\tau}}(\mu) & =\left\{\mu \in I^{X}: \mathfrak{I}_{\tau}(\mu)=1\right\} \\
& =\left\{\mu \in I^{X}: \sup \left\{t \in I_{1}: \mu \wedge t \in \tau\right\}=1\right\} \\
& =\left\{\mu \in I^{X}: \mu \wedge t \in \tau, t<1\right\} \\
& =\tau .
\end{aligned}
$$

and

Conversely, if $\mathfrak{I}$ is a homogeneous $I$-fuzzy topologies on $X$, then $\tau_{\mathfrak{I}}$ is an $I$-topology

$$
\begin{aligned}
\mathfrak{I}_{\mathcal{I}_{\mathfrak{I}}}(\mu) & =\sup \left\{t \in I_{1}: \mu \wedge t \in \tau_{\mathfrak{I}}\right\} \\
& =\sup \left\{t \in I_{1}: \Im(\mu \wedge t)=1\right\} \\
& =1, \quad \text { since } \mathfrak{I}(\mu)=\Im(\mu \wedge t) .
\end{aligned}
$$

So, we are done. 


\section{Connecting $I$-fuzzy Topologies and Preideals}

In this section, we associate for each a fuzzifying topology, a generalized ideal. Also, we construct a preideal corresponding to an $I$-topology, by means of the residuated implication. Conversely, an I-topology, a fuzzifying topology, and I-fuzzy topology are derived by old ones via preideals. We made use of the results of Section 2 , beside the useful following lemma.

Lemma 4.1. Let $\Omega \subseteq I^{X}$. Define $R_{\Omega}, R_{\Omega}^{\prime}: X \times X \rightarrow I$ by for all $(x, y) \in X \times X$,

$$
\begin{aligned}
& R_{\Omega}(x, y)=\inf \left\{\mu(x) \rightarrow \mu(y): \mu \in I^{X}, \mu \in \Omega\right\} \\
& R_{\Omega}^{\prime}(x, y)=\inf \left\{\mu(y) \rightarrow \mu(x): \mu \in I^{X}, 1-\mu \in \Omega\right\}
\end{aligned}
$$

Then $R_{\Omega}, R_{\Omega}^{\prime}$ are fuzzy preorders on $X$, especially, if $*=\min =\wedge$, then for all $(x, y) \in X \times X$,

$$
\begin{aligned}
& R_{\Omega}(x, y)=\inf \{\mu(y): \mu \in \Omega, \mu(x)>\mu(y)\} \\
& R_{\Omega}^{\prime}(x, y)=\inf \{\mu(x): 1-\mu \in \Omega, \mu(x)<\mu(y)\} .
\end{aligned}
$$

Proposition 4.2. Let $\tau$ be an I-topology on a set $X$, and $\Phi$ be a preideal on $X$. Define $\tau^{*}(\Phi) \subseteq I^{X}$, by $\mu \in \tau^{*}(\boldsymbol{\Phi})$ if and only if $\mu \leq \sup \{\lambda \in \tau: \Lambda \wedge(1-\mu) \in \Phi\}$. Then $\tau^{*}(\boldsymbol{\Phi})$ is Chang I-topology on $\mathrm{X}$.

Proof. Direct.

However $\tau^{*}(\boldsymbol{\Phi})$ will be called the I-fuzzy preideal topology. As a special case.

Example 4.3. If $*=\min =\Lambda$, and $\tau=\{\alpha \in I: \alpha$ constans $\}$, then $\tau^{*}(\mathscr{\Xi})=\left\{\mu \in I^{X}: \mu \leq \sup \{\alpha \in\right.$ $I: \alpha \wedge(1-\mu) \in \Phi\}\}$. Consequently, $\tau^{*}(\Phi)$ has the following property: for all $\mu \in I^{X}$ such that $1-\mu \in \Phi$, then $\mu \in \tau^{*}(\boldsymbol{\Phi})$.

Proposition 4.4. Let $\Phi$ be a preideal on a set X. Define $\mathfrak{I}: I^{X} \rightarrow I$ by for all $\mu \in I^{X}, \Im(\mu)=$ $\wedge_{x \in X}(\mu(x) \rightarrow \sup \{\alpha \in I: \alpha \wedge(1-\mu(x)) \in \Phi\})$. Then $\mathfrak{I}$ is a Chang I-fuzzy topology on $X$.

Proof. Clearly, $\Im(1)=\Im(0)=1$, by (I1). Let $\mu, \lambda \in I^{X}$. Then $\Im(\mu \wedge \lambda) \geq \Im(\mu) \wedge \Im(\lambda)$, by (P2) and (I12). Let $\left\{\mu_{i}\right\}_{i \in J} \subseteq I^{X}$. Then $\Im\left(\vee_{i \in J} \mu_{i}\right) \geq \wedge_{i \in J} \Im\left(\mu_{i}\right)$, by (I5) and (P3), so we are done.

The $I$-fuzzy topology $\mathfrak{I}$, given in the above proposition, is called the $I$-fuzzy topology associated with a preideal and denoted by $\Im_{\Phi}$. It is easy to see that $\Im_{\Phi}$ satisfy the following simple properties:

(1) for all $A \subseteq X, \Im_{\Phi}(A)=1$;

(2) for all $\mu \in I^{X}$ and $x \in X$ such that $(1-\mu(x)) \in \Phi$, then $\Im_{\mathscr{\Phi}}(\mu)=1$;

(3) restricting the range of $\mathfrak{I}$ on $\{0,1\}$, a Chang $I$-topology, associated with a preideal $\Phi$, is obtained, namely,

$$
\Im_{\mathscr{\Phi}}=\left\{\mu \in I^{X}: \mu(x) \leq \sup \{\alpha \in I: \alpha \wedge(1-\mu(x)) \in \Phi\}, \forall x \in X\right\} .
$$


Proposition 4.5. Let $\tau$ be an I-topology on a Boolean lattice $X$ with 0 . Let $\Phi \subseteq I^{X}$, defined as $\Phi=\left\{\mu \in I^{X}: \sup _{x \in \mu^{\alpha}} \inf _{\lambda \in \tau}(\lambda(x) \rightarrow \lambda(0)) \leq 1-\alpha\right.$, for all $\left.\alpha \in I_{1}\right\}$. Then $\Phi$ is a saturated preideal on $X$ and $\Phi=\left\{\mu \in I^{X}: d_{R_{\tau}}\left(\mu^{\alpha}\right) \geq \alpha\right.$, for all $\left.\alpha \in I_{1}\right\}$, where $d_{R \tau}$ is the generalized ideal corresponding to $R_{\tau}$.

Proof. Let $\tau$ be an $I$-topology on $X$. Then $R_{\tau}$ is a fuzzy preorder, by Theorem 3.1, hence $d_{R_{\tau}}$ is a generalized ideal, by (2.9) in Proposition 2.6. Thus, $\Phi=\left\{\mu \in I^{X}: d_{R_{T}}\left(\mu^{\alpha}\right) \geq \alpha\right\}$ is a saturated preideal by (2.11) in Proposition 2.7. Secondly, we prove the equality of the two forms of $\Phi$ as follows:

$$
\begin{aligned}
\mathscr{\Phi} & =\left\{\mu \in I^{X}: d_{R_{\tau}}\left(\mu^{\alpha}\right) \geq \alpha\right\} \\
& =\left\{\mu \in I^{X}: \underset{x \in \mu^{\alpha}}{\wedge}\left(1-R_{\tau}(x, 0)\right) \geq \alpha, \forall \alpha \in I_{1}\right\}, \quad \text { by }(2.9) \text { in Proposition } 2.6 \\
& =\left\{\mu \in I^{X}: \underset{x \in \mu^{\alpha}}{\vee} R_{\tau}(x, 0) \leq 1-\alpha, \forall \alpha \in I_{1}\right\} \\
& =\left\{\mu \in I^{X}: \underset{x \notin \mu^{\alpha}}{\vee} \underset{\lambda \in \tau}{\wedge}(\lambda(x) \rightarrow \lambda(0)) \leq 1-\alpha, \forall \alpha \in I_{1}\right\}, \quad \text { by Theorem 3.1, }
\end{aligned}
$$

which completes the proof.

As a direct consequence of Lemma 4.1 and Theorem 3.1, we may construct an Itopology from a preideal, as follows.

Proposition 4.6. Let $\Phi \subseteq I^{X}$ be a preideal. Define $\tau_{\oplus}$ by

$$
\tau_{\Phi}=\left\{\lambda \in I^{X}:\left(\wedge_{\mu \in \Phi} \mu(x) \rightarrow \mu(y)\right) \leq \lambda(x) \rightarrow \lambda(y)\right\}
$$

Then $\tau_{\Phi}$ is an I-Alexandrov topology on $X$, containing $\Phi$.

Proposition 4.7. Let $\Im$ be a nontrivial I-fuzzy topology on a set $X$. Define $\Phi:=\left\{\mu \in I^{X}: \Im(\lambda)=\right.$ 1 , for all $\lambda \leq \mu\}$, then $\Phi$ is a preideal on $X$.

Proof. Straightforward.

Proposition 4.8. Let $\Phi$ be a preideal on a set $X$. Define $\tau \subseteq I^{X}$ by $\tau=\left\{\lambda \in I^{X}: \lambda=\mu \vee \alpha, \mu \in\right.$ $\Phi, \alpha \in I\}$. Then $\tau$ is an I-topology if $\Phi$ is $\sigma$-preideal.

Proof. Since $0 \in \boldsymbol{\Phi}$, then $\tau$ contains all the constants fuzzy subsets on $X$. Secondly, if $\lambda_{1}, \lambda_{2} \in \tau$, then there exist $\mu_{1}, \mu_{2} \in \Phi$ and $\alpha_{1}, \alpha_{2} \in I$ such that $\lambda_{1}=\mu_{1} \vee \alpha_{1}$ and $\lambda_{2}=\mu_{2} \vee \alpha_{2}$, then $\lambda_{1} \wedge \lambda_{2}=$ $\left(\mu_{1} \wedge \mu_{2}\right) \vee\left(\mu_{1} \wedge \alpha_{2}\right) \vee\left(\mu_{2} \wedge \alpha_{1}\right) \vee\left(\alpha_{1} \wedge \alpha_{2}\right)$. By (P3) and (P2), we get $\left(\mu_{1} \wedge \mu_{2}\right) \vee\left(\mu_{1} \wedge \alpha_{2}\right) \vee\left(\mu_{2} \wedge \alpha_{1}\right) \in \Phi$, hence $\lambda_{1} \wedge \lambda_{2} \in \tau$.

Let $\left\{\lambda_{i}\right\}_{i \in J} \subseteq \tau$, then for all $i \in J$, there exists $\mu_{\mathrm{i}} \in \boldsymbol{\Phi}, \alpha_{i} \in I$ such that $\lambda_{i}=\mu_{i} \vee \alpha_{i}$. Therefore, we get $\vee_{i \in J} \lambda_{i}=\left(\vee_{i \in J} \mu_{i}\right) \vee\left(\vee_{i \in J} \alpha_{i}\right) \in \tau$, since $\Phi$ is a $\sigma$-preideal. 
Proposition 4.9. Let $\tau$ be a fuzzifying topology on a Boolean lattice. Define $d: 2^{X} \rightarrow I$ by for all $A \subseteq X, d(A)=\wedge_{x, y \in A} \vee_{B \subseteq X, x, y^{\prime} \in B} \tau(B)$. Then $d$ is a generalized ideal on $X$.

Conversely, let $d$ be a generalized ideal on $X$, then $\tau_{d}: 2^{X} \rightarrow I$, defined by for all $A \subseteq X$,

$$
\tau(A)=\underset{(x, y) \in A \times A^{\prime}}{\wedge} \underset{(x, y) \in X \times B \times B^{\prime}}{\vee} d(B)
$$

is a fuzzifying topology on X.

Proof. It is a direct composition of the two maps defined by Proposition 2.6 and Theorem 3.2. Conversely, follows from Remark 2.12 and Theorem 3.2.

Proposition 4.10. Let $d$ be a $\sigma$-generalized ideal on a set $X$. Define $R_{d}: X \times X \rightarrow I$ by for all $(x, y) \in X \times X, R_{d}(x, y)=\wedge_{(x, y) \in B \times B^{\prime}}(1-d(B))$.

Then, $R_{d}$ is a fuzzy preorder and for all $A \subset X, \tau_{R_{d}}(A)=d(A)$.

Proof. That $R_{d}$ is a fuzzy preorder follows from Remark 2.12, hence according to Theorem 3.2, for all $A \subseteq X$,

$$
\begin{aligned}
\tau_{R_{d}}(A) & =\wedge_{(x, y) \in A \times A^{\prime}}\left(1-R_{d}(x, y)\right) \\
& ={\hat{(x, y) \in A \times A^{\prime}}}^{\wedge}\left(1-{\hat{(x, y) \in B \times B^{\prime}}}^{\wedge}(d(B) \rightarrow 0)\right), \quad \text { by Remark 2.12 } \\
& =\wedge_{(x, y) \in A \times A^{\prime}}\left(\underset{(x, y) \in B \times B^{\prime}}{\vee}(1-(d(B) \rightarrow 0))\right. \\
& \geq 1-(d(A) \rightarrow 0) \\
& \geq 1-(1-d(A))=d(A)) .
\end{aligned}
$$

Secondly, we have to show that for any $A \subseteq X, \tau_{R_{d}}(A) \leq d(A)$.

Let $t \in I_{1}$ such that $\tau_{R_{d}}(A)>t$. Then for any $x \in A, y \in A^{\prime}$, there exists $N_{y}(x) \subseteq X$ such that $x \in N_{y}(x), y \notin N_{y}(x)$, and $d\left(N_{y}(x)\right)>t$. Let $N_{y}=\cup_{x \in A} N_{y}(x)$, then $y \notin N_{y}, A \subseteq N_{y}$, and $d\left(N_{y}\right)=d\left(\cup_{x \in A} N_{y}(x)\right) \geq \wedge_{x \in A} d\left(N_{y}(x)\right) \geq t$, since $d$ is $\sigma$-generelized ideal. Obviously, $A=\cap_{y \notin A} N_{y}$. So we would get $d(A)=d\left(\cap_{y \notin A} N_{y}\right) \geq \vee_{y \notin A} d\left(N_{y}\right) \geq t$, by (G3). Thus $d(A) \geq$ $\tau_{R_{d}}$, which completes the proof.

Example 4.11. (1) Let $\Phi=\left\{\mu \in I^{X}: \mu \leq 1 / 2\right\}$, and $*=$ min, then

$$
x \longrightarrow y= \begin{cases}1, & x \leq y \\ y, & x>y\end{cases}
$$


Then $\Phi$ is a preideal on $X$, and

$$
\begin{aligned}
R_{\Phi}(x, y) & =\wedge_{\mu \in \Phi} \mu(x) \longrightarrow \mu(y), \quad \forall x, y \in X \\
& = \begin{cases}1, & x=y \\
0, & x \neq y\end{cases}
\end{aligned}
$$

is a fuzzy preorder, hence

$$
\tau_{R_{\Phi}}=\left\{\mu \in I^{X}: R_{\Phi}(x, y) \wedge \mu(x) \leq \mu(y), \forall x, y \in X\right\}=I^{I}
$$

Also, another fuzzy preorder $\Re_{\oplus}$ may be dedeuced by $\Phi$, Lemma 4.1 :

$$
\begin{aligned}
\mathfrak{R}_{\Phi}(x, y) & =\inf \{\mu(x): 1-\mu \in \Phi, \mu(x)<\mu(y), \forall x, y \in X\} \\
& =\inf \left\{\mu(x): \mu \in I^{I}, \mu \geq \frac{1}{2}, \mu(x)<\mu(y), \forall x, y \in X\right\} \\
& = \begin{cases}1, & x=y, \\
\frac{1}{2}, & x \neq y .\end{cases}
\end{aligned}
$$

Then

$$
\begin{aligned}
\tau_{\mathfrak{R}_{\Phi}} & =\left\{\lambda \in I^{I}: \mathfrak{R}_{\Phi}(x, y) \wedge \lambda(x) \leq \lambda(y), \forall x, y \in I\right\} \\
& =\left\{\lambda \in I^{I}: \lambda \geq \frac{1}{2}\right\} \bigcup\{\alpha \in I: \alpha \text { constant }\} .
\end{aligned}
$$

(2) Let $\tau=\left\{\mu \in I^{X}: \mu \geq 1 / 2\right\} \cup\{\alpha \in I$ constants $\}$, and $*=\min$, then $\Phi_{\tau}=\left\{\mu \in I^{I}: \mu \leq\right.$ $1 / 2\}$, by applying Proposition 4.5 .

\section{Acknowledgment}

The author is grateful to Professor Etienne Kerre for his warm encouragement.

\section{References}

[1] A. A. Ramadan, M. A. Abdel-Satter, and Y. C. Kim, "Generalized ideals," Journal of Fuzzy Mathematics, vol. 11, no. 1, pp. 67-84, 2003.

[2] M. H. Burton, "The relationship between a fuzzy uniformity and its family of $\alpha$-level uniformities," Fuzzy Sets and Systems, vol. 54, no. 3, pp. 311-315, 1993.

[3] J. Gutiérrez Garcia and M. A. de Prada Vicente, "Super uniform spaces," Quaestiones Mathematicae, vol. 20, no. 3, pp. 291-309, 1997.

[4] R. Lowen, "Fuzzy topological spaces and fuzzy compactness," Journal of Mathematical Analysis and Applications, vol. 56, no. 3, pp. 621-633, 1976. 
[5] P. Venugopalan, "Fuzzy ordered sets," Fuzzy Sets and Systems, vol. 46, no. 2, pp. 221-226, 1992.

[6] A. P. Shostak, "Two decades of fuzzy topology: the main ideas, concepts and results," Russian Mathematical Surveys, vol. 44, no. 6, pp. 125-186, 1989.

[7] R. Lowen, "Fuzzy neighborhood spaces," Fuzzy Sets and Systems, vol. 7, no. 2, pp. 165-189, 1982.

[8] J. Fang and P. Chen, "One-to-one correspondence between fuzzifying topologies and fuzzy preorders," Fuzzy Sets and Systems, vol. 158, no. 16, pp. 1814-1822, 2007.

[9] M. S. Ying, "A new approach for fuzzy topology-I," Fuzzy Sets and Systems, vol. 39, no. 3, pp. 303321, 1991.

[10] H. Lai and D. Zhang, "Fuzzy preorder and fuzzy topology," Fuzzy Sets and Systems, vol. 157, no. 14, pp. 1865-1885, 2006.

[11] J. Fang, "l-fuzzy Alexandrov topologies and specialization orders," Fuzzy Sets and Systems, vol. 158, no. 21, pp. 2359-2374, 2007.

[12] D. Zhang and L. Xu, "Categories isomorphic to FNS," Fuzzy Sets and Systems, vol. 104, no. 3, pp. 373-380, 1999.

[13] P. Wuyts, R. Lowen, and E. Lowen, "Reflectors and coreflectors in the category of fuzzy topological spaces," Computers E Mathematics with Applications, vol. 16, no. 10-11, pp. 823-836, 1988. 


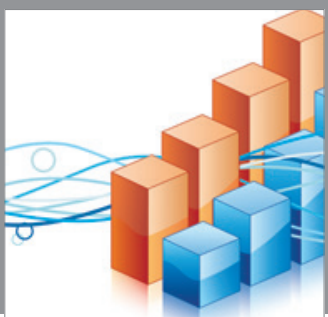

Advances in

Operations Research

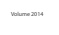

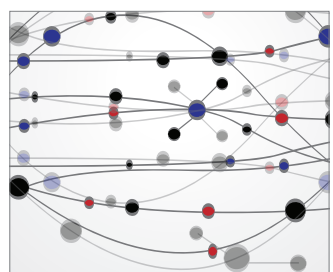

\section{The Scientific} World Journal
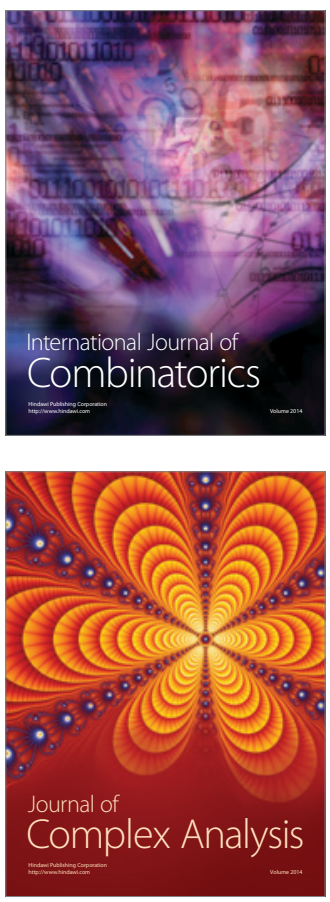

International Journal of

Mathematics and

Mathematical

Sciences
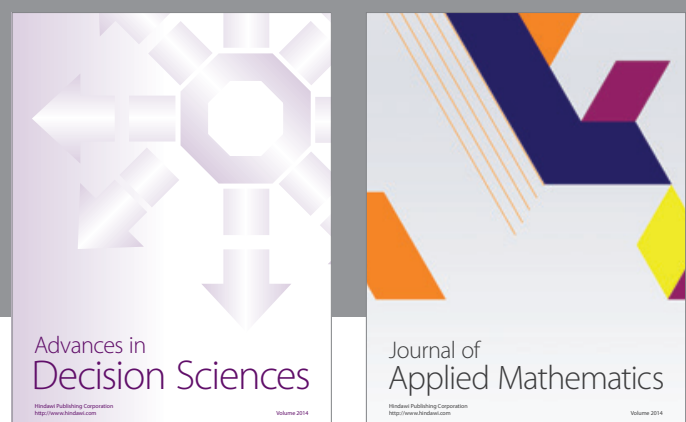

Journal of

Applied Mathematics
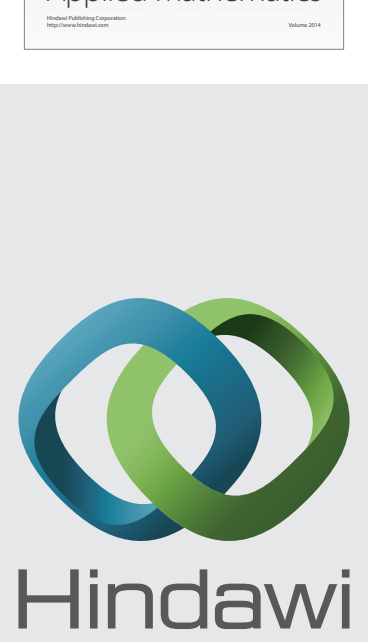

Submit your manuscripts at http://www.hindawi.com
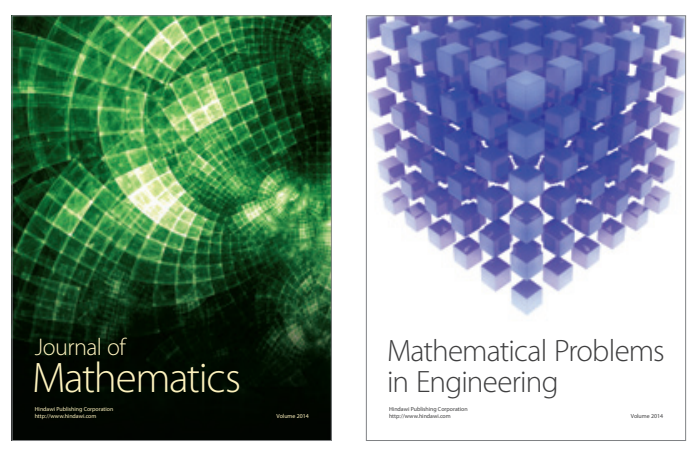

Mathematical Problems in Engineering
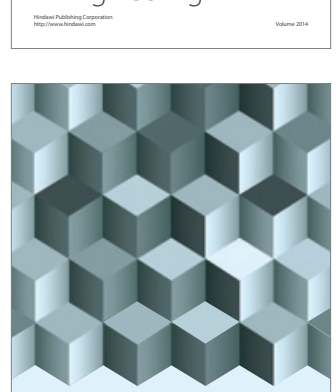

Journal of

Function Spaces
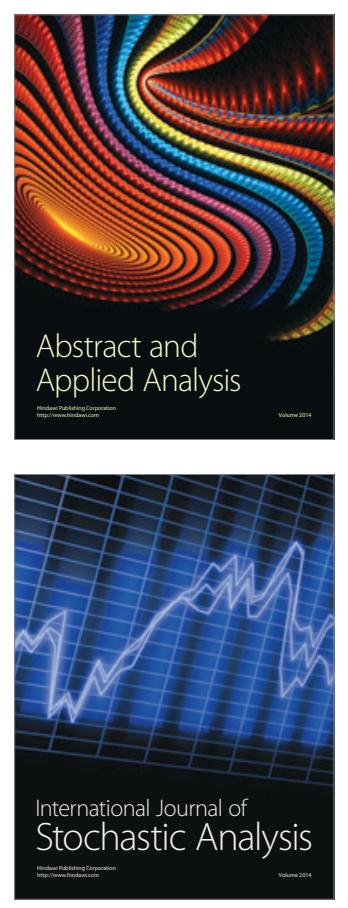

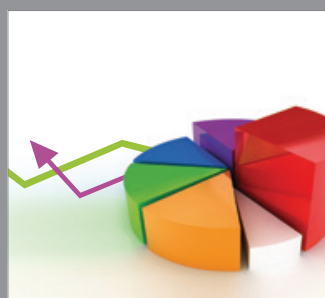

ournal of

Probability and Statistics

Promensencen
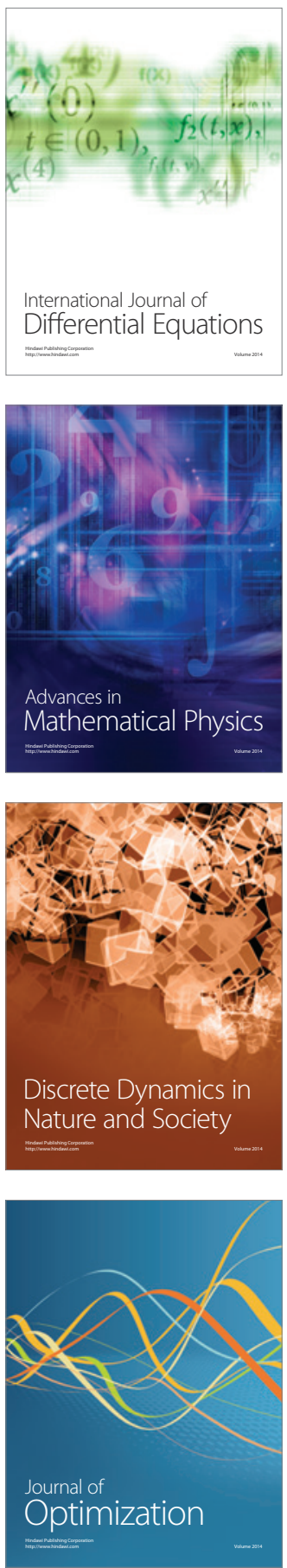